\title{
Mitochondria: multifaceted regulators of aging
}

\author{
Jyung Mean Son $^{1} \mathcal{E}$ Changhan Lee $e^{1,2,3, *}$ \\ ${ }^{1}$ Leonard Davis School of Gerontology, University of Southern California, Los Angeles, CA 90089, ${ }^{2}$ USC Norris Comprehensive Cancer \\ Center, Los Angeles, CA 90089, USA, ${ }^{3}$ Biomedical Science, Graduate School, Ajou University, Suwon 16499, Korea
}

\begin{abstract}
Aging is accompanied by a time-dependent progressive deterioration of multiple factors of the cellular system. The past several decades have witnessed major leaps in our understanding of the biological mechanisms of aging using dietary, genetic, pharmacological, and physical interventions. Metabolic processes, including nutrient sensing pathways and mitochondrial function, have emerged as prominent regulators of aging. Mitochondria have been considered to play a key role largely due to their production of reactive oxygen species (ROS), resulting in DNA damage that accumulates over time and ultimately causes cellular failure. This theory, known as the mitochondrial free radical theory of aging (MFRTA), was favored by the aging field, but increasing inconsistent evidence has led to criticism and rejection of this idea. However, MFRTA should not be hastily rejected in its entirety because we now understand that ROS is not simply an undesired toxic metabolic byproduct, but also an important signaling molecule that is vital to cellular fitness. Notably, mitochondrial function, a term traditionally referred to bioenergetics and apoptosis, has since expanded considerably. It encompasses numerous other key biological processes, including the following: (i) complex metabolic processes, (ii) intracellular and endocrine signaling/communication, and (iii) immunity/inflammation. Here, we will discuss shortcomings of previous concepts regarding mitochondria in aging and their emerging roles based on recent advances. We will also discuss how the mitochondrial genome integrates with major theories on the evolution of aging. [BMB Reports 2019; 52(1): 13-23]
\end{abstract}

\section{INTRODUCTION}

Mitochondria are unique cellular organelles in that they inherently possess their own genome in the form of a circular DNA (mitochondrial DNA; mtDNA) which presumably

*Corresponding author. Tel: +1-310-909-3949; Fax: +1-213-8215885; E-mail: changhan.lee@usc.edu

https://doi.org/10.5483/BMBRep.2019.52.1.300

Received 4 November 2018

Keywords: Aging, Communication, Mitochondria, Mitochondrial DNA, Signaling derived from endosymbiotic alpha-proteobacteria. Traditionally, the main function of mitochondria has been considered to be ATP production by oxidative phosphorylation. However, recently, mitochondria have been increasingly appreciated as a major hub that transmits adaptive regulatory signals to control a wide range of cellular functions, including immunity, survival, and homeostasis, with strong implications in aging. Mitochondria regulate many age-related pathways including senescence, unfolded protein response (UPR), autophagy, and inflammation. Some prominent pathways include the following: (i) reactive oxygen species (ROS) signaling that have a broad cellular impact including nuclear gene regulation, (ii) mitochondrial unfolded response $\left(\mathrm{UPR}^{\mathrm{mt}}\right)$ whereby mitochondrial perturbations activate stress-responsive transcriptional responses in the nucleus via factors such as activating transcription factor associated with stress-1 (ATFS-1) in C. elegans and ATF-5 in mammals, (iii) metabolite signaling, (iv) mitochondrial damage-associated molecular patterns (mtDAMPs) that consist of molecules released from injured mitochondria, and ( $v$ ) mitochondrial-derived peptides (MDPs) that are factors encoded within the mtDNA.

Notably, mitochondrial communication is an emerging biology with increasing evidence for a key role in normal aging and age-related disease, but the mechanistic details are largely unclear. In this review, we will discuss mitochondrial communication, with an emphasis on its influences on cellular function, homeostasis, and aging.

\section{MITOCHONDRIAL GENOMIC INSTABILITY AND AGING}

Several theories have been proposed to unravel the biological basis of aging. The mitochondrial free radical theory of aging (MFRTA) has been a prominent concept that describes mitochondria as a major driving force of aging. First proposed by Denham Harman in the 1950s, the theory posits that the progressive accumulation of cellular damages inflicted by free radicals generated during mitochondrial metabolism leads to aging $(1,2)$. However, MFRTA has been increasingly unfavored because of inconsistent data that suggest alternative mitochondrial contributions to aging. Here, we will discuss the past, present, and future of the role of mitochondria in lifespan and healthspan.

Free radicals are molecules with at least one unpaired 
electron. During mitochondrial oxidative phosphorylation, electrons can 'leak' to form free radicals that react with surrounding oxygen to generate reactive oxygen species (ROS), which in turn can damage cellular macromolecules such as lipids, protein, and DNA. Mitochondrial DNA (mtDNA), due to its proximity to the site of ROS production, was thought to be highly vulnerable (3). In addition, a contemporary notion that the mitochondrial DNA repair system was inferior to the nuclear counterpart provided added support to MRFTA (4). However, mtDNA integrity is maintained at multiple levels, including a repair system that is more versatile than previously thought (5), physical shielding by nucleoids $(6,7)$, mitochondrial fission and fusion $(8,9)$, and mitophagy (10). Nonetheless, mtDNA mutation frequency increases with age in various animal models and humans (11-14), although their role as the driver of aging has been unclear $(15,16)$. A mutation load greater than $60 \%-90 \%$, which is beyond what is incurred by aging, has been suggested to be necessary for age-related phenotypes to manifest (17-19).

Genetic manipulations of the antioxidant system intended to test MFRTA (i.e., the role of ROS in aging) more directly has been inconsistent and inconclusive (20). Only some cellular antioxidant systems that were inactivated shortened lifespan in yeast [i.e. copper-zinc superoxide dismutase (CuZnSOD; sod1), manganese superoxide dismutase (MnSOD; sod2), and copper chaperone (ccs1)] $(21,22)$, worms (sod isoforms) (23), flies (sod1 and sod2) (24-27), and mice (i.e. sod1) (28). Notably, many antioxidant genes did not significantly affect lifespan in these model organisms. On the contrary, it has been shown that overexpression of antioxidant components including sod 1 and sod2 can increase lifespan in yeast (29), worms $(30,31)$, and flies $(32-34)$. In mice, it has been shown that overexpression of human catalase localized to mitochondria (mCAT) can decrease oxidative stress and extend lifespan (35). It can also improve age-dependent insulin resistance (36). One caveat of the report by Schriner et al. (35) was that lifespan extension was significant in mCAT mice that were two to four generations backcrossed to the C57BL/6J strain. However, the longevity effect diminished after $>9$ generations. This could be a secondary effect derived from epistasis and/or CMV element methylation (37).

Perhaps the most direct challenge to MFRTA comes from the failure to detect age-dependent increase in ROS-induced mtDNA damage. DNA mutations that arise from ROS can cause an 8-oxo-2'-deoxyguanosine (8-oxodG)-mediated G-to-T transversion (38). However, mtDNA mutations in brain and heart of old mice ( $>24$ mo. vs $<10$ months) were transitions whereas G-to-T transversions were modest (39). Notably, transitions are mostly caused by replicative infidelity (i.e., DNA polymerase errors), indicating that replicative errors, not ROS, are the main culprit of age-dependent mtDNA mutations. In addition, ultra-deep hepatic mtDNA sequencing showed increased age-dependent replicative errors, not ROS-dependent damage (40). Similarly, highly sensitive duplex sequencing of aged human pre-frontal cortex mtDNA ( $>75 \mathrm{yrs}$ vs. $<1 \mathrm{yr}$ ) revealed higher proportions of replication errors rather than oxidative damage (41).

Inactivating the proofreading activity of mitochondrialspecific DNA polymerase $\gamma$ (mtDNA mutator in mice) by targeted mutagenesis at amino acid position 257 (D257A) increased mtDNA mutation frequency to supraphysiological levels in mice: $\sim 2,500$-fold and $\sim 500$-fold higher in homozygous (polg ${ }^{\text {mutmut }}$ ) and heterozygous (polg $\left.{ }^{+/ m u t}\right)$ mutant mice, respectively (39). Although homozygous (polgmutmut) mice exhibited accelerated aging phenotypes and significantly reduced lifespan, heterozygous ( polg $^{+/ m u t}$ ) mice did not show early signs of aging. They had a normal lifespan (39, 42). Furthermore, mtDNA mutator mice exhibited OXPHOS dysfunction without significant increase of oxidative damage (43-45). Notably, ROS levels in young mtDNA mutator mice were not increased despite high levels of mtDNA mutations (46). Nonetheless, these mutations have been implicated in more than 300 diseases that are linked to aging and age-related diseases listed in the Human DNA Polymerase Gamma Mutation Database (http://tools.niehs.nih.gov/polg) (47). Lastly, mtDNA deletions that become prevalent with aging (48-50) are significantly increased in short-lived homozygous (polg ${ }^{\text {mutmut }}$ ) mice, but not in heterozygous (polg ${ }^{+/ m u t}$ ) mice that had a normal lifespan (51). These results suggest a more complicated connection between mtDNA mutation frequency and aging. Further investigations are needed to identify other aspects of mtDNA mutator mice such as mitochondrial communication.

\section{CELL-AUTONOMOUS MITOCHONDRIAL COMMUNICATION AND AGING}

Eukaryotic cells are functionally compartmentalized into organelles with assigned distinct tasks that work in concert. Such subcellular coordination is mediated by inter-organellar communication to maintain cellular homeostasis. The mechanism underlying inter-organellar communication is an emerging topic in biology that has much implications for aging. On that line, the connection between mitochondria and the nucleus is of special interest considering that they uniquely possess independent genomes (Fig. 1A).

\section{Mitonuclear communication}

Mitochondria presumably originate from $\alpha$-proteobacteria that have sustained an endosymbiotic relationship with our ancestral cells $\sim 1.5$ billion years ago. Notably, mitochondrial retained a portion of the original bacterial genomes that co-evolved with nuclear genome. However, mitochondria import over a thousand proteins encoded in the nuclear genome to maintain their diverse functions, reflecting their close relationship. Therefore, it is critical that mitochondria and the nucleus dynamically communicate (i.e., mitonuclear 
A

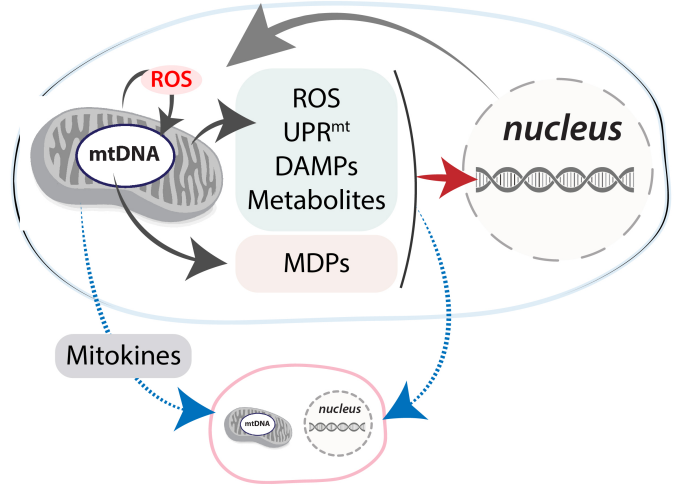

B

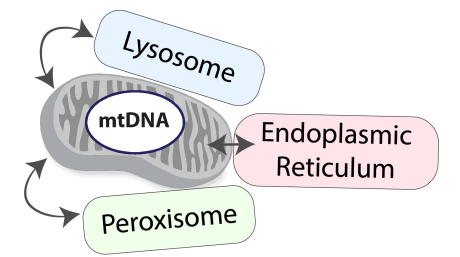

Fig. 1. Mitochondrial Communication Modes. (A) Mitochondria communicate to the nucleus and other cells (i.e., mitochondrial endocrine signaling) using various mediators such as ROS, UPR $^{\mathrm{mt}}$, DAMPs, and mitochondrialencoded MDPs. (B) Mitochondria also communicate with other organelles (e.g., endoplasmic reticulum, lysosomes, and peroxisomes) to coordinate complex cellular processes. communication) with each other to coordinate adaptive responses to the constantly changing intrinsic and extrinsic cellular milieu and maintain homeostasis. In fact, impaired mitonuclear communication is strongly implicated in aging and age-dependent diseases. Mitonuclear communication is bi-directional. It is mediated by several factors transmitted from each organelle. In this review, we will focus on signals transmitted from mitochondria to the nucleus (i.e., retrograde signals), including reactive oxygen species (ROS), mitochondrial unfolded protein response (UPR $\left.{ }^{\mathrm{mt}}\right)$, metabolites, mitochondrial damage-associated molecular patterns (mtD AMP), and mitochondrial derived peptides (MDPs).

\section{Reactive Oxygen Species (ROS) signaling}

ROS is often considered as a toxic metabolic byproduct that causes detrimental damage to multiple cellular components, thereby contributing to the aging process $(3,52)$. However, as discussed above, mitochondrial ROS is not the major cause of mtDNA mutations, indicating a more complex cellular role. Actions of ROS are pleiotropic. They cause oxidative stress at higher concentrations (pathological) while they act as signaling molecules at lower levels (physiological). In fact, physiological ROS response has been suggested as eustress (53) that increases mitohormesis as an adaptive response to promote health and extend lifespan (54). Increasing interest in ROS as signaling molecules that regulate normal physiological processes has provided insight into its role in regulating lifespan and/or healthspan (53, 55, 56). In fact, lifespan extension in various model organisms such as worm, fly, and mice is mediated by retrograde ROS. In worms, RNAi screens have identified long-lived animals that harbor mutations in mitochondrial respiration $(57,58)$. On that line, retrograde ROS signaling can mediate lifespan extension in worms with impaired insulin/IGF-1 signaling while inhibition of ROS signals using antioxidants can reduce such longevity by up to $60 \%$ (59). Furthermore, a mild increase in ROS levels by inhibiting respiration can activate transcription factor hypoxia-inducible factor 1 (HIF-1) and consequent nuclear gene expression to promote longevity in worms (60). In addition to modulating factors, ROS can also lead to epigenetic alternations $(61,62)$. For instance, ROS can regulate the chromatin binding capacity of histone demethylase Rph1p, thereby extending chronological lifespan in yeast (63). Moreover, ROS can promote mitochondrial unfolded protein response $\left(\mathrm{UPR}^{\mathrm{mt}}\right)$. This will be further discussed in the following section.

\section{Mitochondrial unfolded protein response (UPR ${ }^{\mathrm{mt}}$ )}

Mitochondrial unfolded protein response $\left(U P R^{\mathrm{mt}}\right)$ is a mitochondria-to-nuclear communication mechanism that promotes adaptive regulation of nuclear genes related to mitochondrial chaperones, proteases, antioxidants, xenobiotic response, and metabolism, ultimately rewiring the cell to survive. UPR ${ }^{\mathrm{mt}}$ was initially thought to be triggered by $\mathrm{mtDNA}$ depletion or by protein misfolding in the mitochondrial matrix (64). However, it now encompasses various mitochondrial stress conditions, including dysfunctional metabolism, defective iron sulfur cluster assembly, and immune response $(64,65)$. The activating transcription factor associated with stress 1 (ATFS-1) in worms is a major mediator of UPR ${ }^{\mathrm{mt}}$. Normally, ATFS-1 is imported into mitochondria for proteolytic degradation. However, mitochondrial stress will trigger ATFS-1 to translocate from mitochondria to the nucleus where it regulates the expression of a considerable portion of mitochondrial stress-responsive genes $(65,66)$. Such bi-organellar trafficking to coordinate mitonuclear communication is possible because ATFS-1 possesses both a mitochondrialtargeting sequence (MTS) and a nuclear localization signal (NLS) $(65,66)$. ATFS-1 also plays a role in chromatin remodeling which is required for full activation of UPR ${ }^{\mathrm{mt}}$ via the histone methyltransferase met- 2 and a nuclear co-factor lin-65 to promote longevity (67). CLOCK-1 (CLK-1; human homolog COQ7) acts as a ROS barometer that mediates mitochondria to nuclear signaling by activating UPR ${ }^{\mathrm{mt}}$. clk-1 null worms have extended lifespans (68). Such effect appears to be mediated by UPR ${ }^{\mathrm{mt}}(66)$. In mice, the loss of clk1 also 
increases cellular fitness and lifespan (69).

\section{Metabolite signaling}

Mitochondria are metabolic hubs that perform a wide range of catabolic and anabolic processes, thereby generating a variety of metabolites. Mitochondrial metabolites can also act as secondary messengers for genetic or epigenetic regulation (70, 71). Of these metabolites, many are products of the tricarboxylic acid (TCA) cycle, such as acetyl-coenzyme A (acetyl-CoA), succinyl-CoA, and nicotinamide adenine dinucleotide $\left(\mathrm{NAD}^{+}\right)$. The pyruvate dehydrogenase $(\mathrm{PDH})$ complex that normally resides in mitochondria and generates acetyl-coenzyme A (acetyl-CoA) can translocate from mitochondria to the nucleus where it is involved in producing acetyl-CoA in the nucleus and modulate histone acetylation which requires acetyl-CoA as a substrate for lysine acetylation $(72,73)$. Under growth conditions, acetyl-CoA levels are higher in the nucleus and cytosol for lipid synthesis and histone acetylation. However, under starvation conditions, acetyl-CoA predominantly resides in mitochondria for ATP and ketone body production (74). Succinyl-CoA is another TCA cycle intermediate that can post-translationally modify proteins by succinylating lysine residues of proteins (75) such as histones, thereby affecting chromatin dynamics and consequently the epigenome (76). In a similar way, other TCA intermediates, including oxaloacetate, malate, fumarate and $\alpha$-ketoglutarate, can also induce genetic and epigenetic reprogramming and extend worm lifespan (77). $\mathrm{NAD}^{+}$is also a crucial mitochondrial gero-metabolite that declines with age $(78,79)$. Reduced age-dependent $\mathrm{NAD}^{+}$availability is linked to decreased deacetylase sirtuin activities, ultimately affecting the communication between mitochondria and nucleus (80, 81). It also affects longevity $(82,83)$. Retrograde $\mathrm{Ca}^{2+}$ is another important inorganic gero-metabolite (84). Nuclear skeletal muscle gene expression is regulated by mitochondrial $\mathrm{Ca}^{2+}$ which mediates mitochondria to nucleus route (85).

\section{Mitochondrial damage-associated molecular patterns (mtDAMP)}

Our immune system becomes progressively impaired with age, leading to the loss of immune function (i.e., immunosenescence) and elevated chronic low-grade inflammation (i.e., inflammaging) (86). Immune responses can be triggered not only by foreign materials/organisms, but also by endogenous factors. Pathogen-associated molecular patterns (PAMPs) derived from bacteria, fungi, and viruses can induce innate immune responses via inflammasomes that are intracellular complexes capable of promoting pro-inflammatory cytokines such as interleukin-1 $\beta$ (IL-1 $\beta$ ) and IL-18 (87). Damage-associated molecular patterns (DAMPs) derived from endogenous intracellular components that are released during cellular stress and/or damage can also mount an immune response (88). Especially, injured mitochondria can release their contents known as mitochondrial damage-associated molecular patterns (mtDAMP) recognized as PAMPs owing to their bacterial ancestry. Some well described mtDAMPs include mtDNA, $\mathrm{N}$-formyl peptides (specific to mitochondriallytranslated proteins), and fragments of mitochondrial proteins $(89,90)$. The innate immune system can express proinflammatory cytokines upon sensing circulating mtDNA and $\mathrm{N}$-formyl peptides using pattern recognition receptors (PRR) such as toll-like receptors (TLRs) and NOD-like receptors (NLRs) (90). Notably, circulating mtDNA levels are increased with age. Their increase is associated with elevated levels of cytokines and inflammatory markers, indicating a role of mtDNA in inflammaging (91) and may contribute to the development of age-related diseases $(92,93)$.

\section{Mitochondrial-derived peptides (MDPs)}

The human mtDNA encodes only 13 protein-coding genes that are all structural components of the electron transport chain (ETC) without known signaling roles. Thus, active geneencoded mitonuclear communication pathways were known to be exclusively mediated by factors encoded in the nuclear genome. More recently, short open reading frames (sORFs) encoded in the mitochondrial genome that yield bioactive peptides, collectively referred to as mitochondrial-derived peptides (MDPs), have been identified (94). There are now eight published MDPs, including humanin, MOTS-C (mitochondrial open reading frame of the twelve S rRNA type-c), and small humanin-like peptide (SHLP) 1-6. They regulate various cellular functions. Humanin is encoded within the mitochondrial $16 \mathrm{~S}$ rRNA. It was identified from a surviving brain fraction of an Alzheimer's disease (AD) patient as a protective factor against AD-related toxins such as $\beta$-amyloid (95). It is also a binding partner of insulin-like growth factor binding protein 3 (IGFBP-3) (96) and an anti-apoptotic factor that inhibits Bax (97). SHLP 1-6 were also identified within the 16S rRNA (98). MOTS-c is encoded within the mitochondrial $12 \mathrm{~S}$ rRNA. It acts as a regulator of metabolic homeostasis that can prevent diet-induced obesity and insulin resistance, and age-dependent insulin resistance in mice (99-101). Notably, MOTS-c can translocate to the nucleus upon cellular stress to regulate adaptive nuclear gene expression by interacting with other stress-responsive transcription factors including nuclear factor erythroid 2-related factor 2 (NFE2L2/NRF2) and binding to chromatin (102-104). HEK293 cells that over-express MOTS-c were significantly protected against metabolic stress (i.e., glucose and serum deprivation) (102). This indicates that our co-evolved mitonuclear genomes have established a genetically integrated bi-directional communication system.

Humanin, SHLP2, and MOTS-c levels decline with age and their actions are positively correlated with longevity $(94,98$, $100,105)$. Humanin levels are negatively regulated by the $\mathrm{GH} / \mathrm{IGF}$ axis in both mice and humans (106). Circulating humanin levels are elevated in long-lived $\mathrm{GH}$-deficient Ames mice, but decreased in short-lived GH-transgenic mice (106). 
MOTS-c can reverse age-dependent insulin resistance. A functional MOTS-c polymorphism is related to exceptional longevity in a Japanese population $(107,108)$. Furthermore, MOTS-c actions are, in part, dependent on sirtuin 1 (SIRT1) and AMPK $(100,102)$, two prominent related factors shown to regulate longevity in various model organisms $(109,110)$.

Because of the unique bi-genomic cellular setup, it is important to consider mitonuclear epistasis. mtDNA is maternally transmitted, which forces a cell to coordinate gene expression with a foreign (i.e., paternal) genome upon fertilization. The compatibility between maternal mtDNA and paternal nuclear DNA is a major ground for intergenomic epistasis (111-113). Nuclear gene expression is dependent on the mtDNA background. Nuclear mutations can manifest very differently under varying mtDNA context. For instance, patients with a mutation in the adenine nucleotide translocator 1 gene (SLC25A4, ANT1) exhibit a wide range of cardiomyopathies that are correlated with their mtDNA lineage (114). In 2015, the United Kingdom approved mtDNA replacement therapy to allow a woman to transfer her nuclear genome to an egg with healthy mitochondria to prevent transmission of mtDNA disease (so-called three-parent baby). Such forced mtDNA-nDNA combinations may be incompatible. It can cause dysregulated mitonuclear communication (Hamilton 2015). In fact, alloantigenicity and immune rejection have been documented in nuclear-transfer-derived embryonic stem cells (NT-ESCs) (115). In addition, Mitochondrial-Nuclear eXchange (MNX) mice with interchanged nuclear and mitochondrial genomes from different mice (similar to three-parent baby) have differential oxidative stress, resistance to heart failure, lipid concentration, and bioenergetics $(116,117)$. Aging is a complex process with strong genetic components. It is likely to be dependent on both of our genomes. Thus, the interaction between factors encoded in each genome may further our understanding of aging genetics.

\section{Other mito-organellar communication}

Cellular functions are compartmentalized into various organelles with unique roles. Their orchestrated processes together support survival. Therefore, inter-organellar communication is key to cellular homeostasis and ultimately organismal fitness. On that line, mitochondria not only communicate with the nucleus, but also dynamically interact with other organelles (118) (Fig. 1B). Although the field of inter-organellar communication is still in its early stages, it is undoubtedly of great interest. Further investigation of mito-organellar communication with high spatial and temporal resolution and identification of key signaling mediators are necessary to understand the complex coordination of subcellular processes and their roles in aging. Here, we will focus on mitochondrial communication with the ER, peroxisomes, and lysosomes in the context of aging.

\section{Mitochondria and Endoplasmic Reticulum (ER) Communication}

The ER physically interacts with mitochondria to regulate organelle morphology and various metabolic signaling (119). The contact sites that ER forms with mitochondria are called mitochondria-associated membranes (MAM), which have numerous roles in controlling lipid and calcium homeostasis, mitochondrial metabolism, insulin and glucose signaling, and ultimately aging (120). Proteome analysis of MAM has revealed its connection to various age-related diseases, such as Alzheimer's disease and type 2 diabetes (120). Cisd2 knockout mice also provide evidence that MAM may play a role in aging. Cisd2 is a regulator of intracellular $\mathrm{Ca}^{2+}$ and glucose homeostasis that localizes to the $E R$, mitochondrial membranes, and MAM (121). Mice that lacked Cisd2 showed mitochondrial degeneration and functional decline in skeletal muscle and neurons, glucose intolerance, premature aging phenotypes (e.g. ocular degeneration, dermal deterioration, sarcopenia, etc.), and shortened lifespan (122), implying that disruption of mitochondria and ER communication could affect the aging process.

\section{Mitochondria and Peroxisome Communication}

Increasing evidence points to the mitochondrial-peroxisomal connection as an important aspect of aging and age-related disease $(123,124)$. Restoring the import of peroxisomal catalase which decomposes hydrogen peroxide can restore mitochondrial integrity and reverse the senescent phenotype of human fibroblasts (125). Another study demonstrated that peroxisome proliferation and higher peroxisomal antioxidant activity can regulate the aging of hippocampal neurons (126, 127). Furthermore, the three-way communication among mitochondria, peroxisome, and ER may contribute to the aging process by fine-tuning redox and ion signaling pathways (128). Redox-regulatory enzymes can assemble at the "redox triangle" created by these three organelles to sense ROS accumulations and redox imbalances. The redox triangle may become dysfunctional with age (128). However, further investigations on the mechanistic details regarding the multidirectional communication among mitochondria, ER, and peroxisome and their roles in aging are needed.

\section{Mitochondria and Lysosome Communication}

Lysosomes are subcellular sites of protein turnover and metabolite storage. Its dysfunction is linked to aging and age-associated diseases (129). Mito-lysosomal communication is mediated by physical contact, lipids, and metabolite exchange (130). In yeast, lysosome-like vacuoles are functionally linked to mitochondria. Increased vacuolar $\mathrm{pH}$ gives rise to age-dependent mitochondrial dysfunction (131), indicating that mito-lysosomal communication is important for organismal homeostasis and lifespan. In addition, the contact site between mitochondria and yeast lysosome-like vacuoles 
known as VCALMP (vacuole and mitochondria patch) is enriched with ion and amino acid transporters. It is important for lipid exchange between the two organelles (132). Furthermore, the inter-organellar lipid homeostasis coordinated among mitochondria, lysosomes, and ER [ER-mitochondria encounter structure (ERMES)) may be important in aging (132, 133). This is also supported by Ltc1 (lipid transfer at contact site 1), a sterol-dependent regulator of organelle and cellular homeostasis via its dual localization to ER-mitochondria and ER-vacuole contact sites (134). It is especially important in nutrient sensing and signaling (135) as well as replicative aging in yeast (131).

\section{NON-CELL AUTONOMOUS MITOCHONDRIAL COMMUNICATION AND AGING}

Mitochondrial communication is not confined to intracellular coordination. Recent studies have shown that mitochondria can also transmit signals to distal cells of different tissues as described in this section. Such non-cell autonomous mitochondrial signals are often referred as mitochondrial cytokines (mitokines) or mitochondrial hormones. The evolutionary aspect of intra-organ mitochondrial communication is interesting in that they may represent an archaic endocrine system. Non-cell autonomous mitochondrial signals provide another layer of endocrine regulation of longevity (Fig. 1A).

\section{Mitokines}

The connection between UPR ${ }^{\mathrm{mt}}$ and longevity has been investigated in mutant worms with perturbed mitochondrial ETC (e.g., cco-1 knockdown) that exhibited extended lifespan (136). Interestingly, cco-1 knockdown in neurons activated $\mathrm{UPR}^{\mathrm{mt}}$ in the intestine, indicating a soluble factor that could relay signals between distal tissues, dubbed mitokine (136, 137). In addition, Wnt signaling may be a mitokine in worms (138). Neuronal expression of the Wnt ligand/EGL-20 in worms activated cell-non-autonomous $\mathrm{UPR}^{\mathrm{mt}}$ that required orchestrated actions of a retromer complex, Wnt signaling, and serotonin (138). In flies, mild ETC disruption in muscles prolonged lifespan through UPR ${ }^{\mathrm{mt}}$ and insulin signaling (140). In mice, fibroblast growth factor 21 (Fgf21) has also been proposed as a mitokine because its production by muscle cells can trigger mitochondrial biogenesis, browning of white adipose tissue (WAT), and increase lipid oxidation (140). These results support the existence of systemic mitochondrial communication factors that can regulate longevity, including neurotransmitters and neuropeptides $(141,142)$. The scope of factors that can act as mitokines is likely to be broad.

\section{Mitochondrial derived peptides (MDPs)}

MDPs are found in circulation. They can act on certain tissues. Thus, they have been dubbed mitochondrial hormones (84, 143, 144). Circulating humanin levels are decreased with age in mice and humans $(105,106,145)$. Humanin is integrated with the GH/IGF-1 axis which is the most prominent endocrine regulator of aging. Long-lived $\mathrm{GH}$-deficient Ames mice showed higher circulating humanin levels whereas short-lived GH-transgenic mice had lower humanin levels compared to their wild type counterparts (106). Notably, an Ecuadorian cohort with $\mathrm{GH}$ receptor deficiency (GHRD) that have very low levels of IGF-I are exceptionally protected against cancer and diabetes (146). However, they showed $80 \%$ increase in circulating humanin levels compared to their unaffected relatives. These studies indicate a role for humanin as an endocrine regulator of aging that is tethered with the $\mathrm{GH} / \mathrm{IGF}-1$ axis. Similar to humanin, levels of circulating SHLP2 are also decreased with age, indicating its relevance to aging as a mitochondrial hormone (147). Plasma levels of MOTS-c are also decreased $\sim 30 \%$ in old mice. Systemic injection of MOTS-c reversed age-dependent skeletal muscle insulin resistance in mice (100).

\section{CONCLUSION}

Mitochondria are versatile organelles that play roles in multiple cellular functions that ultimately affect organismal fitness and lifespan/healthspan. The multifaceted nature of mitochondria indicates its complex roles in aging and age-related diseases. Thus, it is imperative to investigate how mitochondria contribute, and even drive, aging with a comprehensive and holistic approach. The silver lining of the downfall of MFRTA is that dynamic expansion of concepts and experimental data have continued to reveal the complexity and breadth of mitochondria in aging and age-related diseases.

\section{ACKNOWLEDGEMENTS}

This work was funded by grants from NIH (R01AG052558), the Ellison Medical Foundation, the American Federation for Aging Research (AFAR), and the Hanson-Thorell family to C.L., and an American Federation for Aging Research (AFAR) fellowship to J.M.S.

\section{CONFLICTS OF INTEREST}

C.L. is a consultant for and a shareholder of CohBar, Inc. The remaining authors declare no competing interests.

\section{REFERENCES}

1. Harman D (1956) Aging: a theory based on free radical and radiation chemistry. J Gerontol 11, 298-300

2. Harman D (2009) Origin and evolution of the free radical theory of aging: a brief personal history, 1954-2009. Biogerontology 10, 773

3. Harman D (1972) The biologic clock: the mitochondria? J Am Geriatr Soc 20, 145-147

4. Yakes FM, Van Houten B (1997) Mitochondrial DNA damage is more extensive and persists longer than 
nuclear DNA damage in human cells following oxidative stress. Proc Natl Acad Sci U S A 94, 514-519

5. Kazak L, Reyes A, Holt IJ (2012) Minimizing the damage: repair pathways keep mitochondrial DNA intact. Nat Rev Mol Cell Biol 13, 659-671

6. Kang D, Kim SH, Hamasaki N (2007) Mitochondrial transcription factor A (TFAM): roles in maintenance of mtDNA and cellular functions. Mitochondrion 7, 39-44

7. Lee SR, Han J (2017) Mitochondrial nucleoid: shield and switch of the mitochondrial genome. Oxid Med Cell Longev 2017 [Epub ahead of print]

8. Chen H, Vermulst M, Wang YE et al (2010) Mitochondrial Fusion Is Required for mtDNA Stability in Skeletal Muscle and Tolerance of mtDNA Mutations. Cell 141, 280-289

9. Prevost CT, Peris N, Seger C et al (2018) The influence of mitochondrial dynamics on mitochondrial genome stability. Curr Genet 64, 199-214

10. Pickles S, Vigié P, Youle RJ (2018) Mitophagy and Quality Control Mechanisms in Mitochondrial Maintenance. Curr Biol 28, R170-R185

11. Cortopassi GA, Arnheim N (1990) Detection of a specific mitochondrial DNA deletion in tissues of older humans. Nucleic Acids Res 18, 6927-6933

12. Piko L, Hougham AJ, Bulpitt KJ (1988) Studies of sequence heterogeneity of mitochondrial DNA from rat and mouse tissues: evidence for an increased frequency of deletions/additions with aging. Mech Ageing Dev 43, 279-293

13. Larsson NG (2010) Somatic mitochondrial DNA mutations in mammalian aging. Annu Rev Biochem 79, 683-706

14. Payne BA, Wilson IJ, Yu-Wai-Man P et al (2013) Universal heteroplasmy of human mitochondrial DNA. Hum Mol Genet 22, 384-390

15. Linnane AW, Marzuki S, Ozawa T, Tanaka M (1989) Mitochondrial DNA mutations as an important contributor to ageing and degenerative diseases. Lancet 1, 642-645

16. Khrapko K, Vijg J (2009) Mitochondrial DNA mutations and aging: devils in the details? Trends Genet 25, 91-98

17. Rossignol R, Faustin B, Rocher C, Malgat M, Mazat JP, Letellier T (2003) Mitochondrial threshold effects. Biochem J 370, 751-762

18. Stewart JB, Chinnery PF (2015) The dynamics of mitochondrial DNA heteroplasmy: implications for human health and disease. Nat Rev Genet 16, 530-542

19. Kauppila TES, Kauppila JHK, Larsson NG (2017) Mammalian Mitochondria and Aging: An Update. Cell Metab 25, 57-71

20. Pomatto LCD, Davies KJA (2018) Adaptive homeostasis and the free radical theory of ageing. Free Radic Biol Med 124, 420-430

21. Unlu ES, Koç A (2007) Effects of deleting mitochondrial antioxidant genes on life span. Ann N Y Acad Sci 1100, 505-509

22. Longo VD, Gralla EB, Valentine JS (1996) Superoxide dismutase activity is essential for stationary phase survival in Saccharomyces cerevisiae Mitochondrial production of toxic oxygen species in vivo. J Biol Chem
271, 12275-12280

23. Doonan R, McElwee JJ, Matthijssens F et al (2008) Against the oxidative damage theory of aging: superoxide dismutases protect against oxidative stress but have little or no effect on life span in Caenorhabditis elegans. Genes Dev 22, 3236-3241

24. Kirby K, Hu J, Hilliker AJ, Phillips JP (2002) RNA interference-mediated silencing of Sod2 in Drosophila leads to early adult-onset mortality and elevated endogenous oxidative stress. Proc Natl Acad Sci U S A 99, 16162-16167

25. Martin I, Jones MA, Rhodenizer D et al (2009) Sod2 knockdown in the musculature has whole-organism consequences in Drosophila. Free Radic Biol Med 47, 803-813

26. Duttaroy A, Paul A, Kundu M, Belton A (2003) A Sod2 null mutation confers severely reduced adult life span in Drosophila. Genetics 165, 2295-2299

27. Wicks S, Bain N, Duttaroy A, Hilliker AJ, Phillips JP (2009) Hypoxia rescues early mortality conferred by superoxide dismutase deficiency. Free Radic Biol Med 46, 176-181

28. Pérez VI, Bokov A, Van Remmen $\mathrm{H}$ et al (2009) Is the oxidative stress theory of aging dead? Biochim Biophys Acta 1790, 1005-1014

29. Fabrizio P, Liou LL, Moy VN et al (2003) SOD2 functions downstream of Sch9 to extend longevity in yeast. Genetics 163, 35-46

30. Cabreiro F, Ackerman D, Doonan R et al (2011) Increased life span from overexpression of superoxide dismutase in Caenorhabditis elegans is not caused by decreased oxidative damage. Free Radic Biol Med 51, 1575-1582

31. Melov S, Ravenscroft J, Malik S et al (2000) Extension of Life-Span with Superoxide Dismutase/Catalase Mimetics. Science 289, 1567-1569

32. Curtis C, Landis GN, Folk D et al (2007) Transcriptional profiling of MnSOD-mediated lifespan extension in Drosophilareveals a species-general network of aging and metabolic genes. Genome Biol 8, R262

33. Sun J, Folk D, Bradley TJ, Tower J (2002) Induced overexpression of mitochondrial Mn-superoxide dismutase extends the life span of adult Drosophila melanogaster. Genetics 161, 661-672

34. Parkes TL, Elia AJ, Dickinson D, Hilliker AJ, Phillips JP, Boulianne GL (1998) Extension of Drosophila lifespan by overexpression of human SOD1 in motorneurons. Nat Genet 19, 171-174

35. Schriner SE, Linford NJ, Martin GM et al (2005) Extension of murine life span by overexpression of catalase targeted to mitochondria. Science 308, 19091911

36. Lee HY, Choi CS, Birkenfeld AL et al (2010) Targeted expression of catalase to mitochondria prevents age-associated reductions in mitochondrial function and insulin resistance. Cell Metab 12, 668-674

37. Brooks AR, Harkins RN, Wang P, Qian HS, Liu P, Rubanyi GM (2004) Transcriptional silencing is associated with extensive methylation of the CMV promoter following adenoviral gene delivery to muscle. J 
Gene Med 6, 395-404

38. Cooke MS, Evans MD, Dizdaroglu M, Lunec J (2003) Oxidative DNA damage: mechanisms, mutation, and disease. FASEB J 17, 1195-1214

39. Vermulst M, Bielas JH, Kujoth GC (2007) Mitochondrial point mutations do not limit the natural lifespan of mice. Nat Genet 39, 540-543

40. Ameur A, Stewart JB, Freyer C et al (2011) Ultra-deep sequencing of mouse mitochondrial DNA: mutational patterns and their origins. PLoS Genet 7, e1002028

41. Kennedy SR, Salk JJ, Schmitt MW, Loeb LA (2013) Ultra-sensitive sequencing reveals an age-related increase in somatic mitochondrial mutations that are inconsistent with oxidative damage. PLoS Genet 9, e1003794

42. Trifunovic A, Wredenberg A, Falkenberg M et al (2004) Premature ageing in mice expressing defective mitochondrial DNA polymerase. Nature 429, 417-423

43. Edgar D, Shabalina I, Camara Y et al (2009) Random point mutations with major effects on protein-coding genes are the driving force behind premature aging in mtDNA mutator mice. Cell Metab 10, 131-138

44. Kujoth GC, Hiona A, Pugh TD et al (2005) Mitochondrial DNA Mutations, Oxidative Stress, and Apoptosis in Mammalian Aging. Science 309, 481-484

45. Trifunovic A, Hansson A, Wredenberg A et al (2005) Somatic mtDNA mutations cause aging phenotypes without affecting reactive oxygen species production. Proc Natl Acad Sci U S A 102, 17993-17998

46. Logan A, Shabalina IG, Prime TA et al (2014) In vivo levels of mitochondrial hydrogen peroxide increase with age in mtDNA mutator mice. Aging Cell 13, 765-768

47. DeBalsi KL, Hoff KE, Copeland WC (2017) Role of the mitochondrial DNA replication machinery in mitochondrial DNA mutagenesis, aging and age-related diseases. Ageing Res Rev 33, 89-104

48. Bua E, Johnson J, Herbst A et al (2006) Mitochondrial DNA-Deletion Mutations Accumulate Intracellularly to Detrimental Levels in Aged Human Skeletal Muscle Fibers. Am J Hum Genet 79, 469-480

49. Cortopassi GA, Arnheim N (1990) Detection of a specific mitochondrial DNA deletion in tissues of older humans. Nucleic Acids Res 18, 6927-6933

50. Bender A, Krishnan KJ, Morris CM et al (2006) High levels of mitochondrial DNA deletions in substantia nigra neurons in aging and Parkinson disease. Nat Genet 38, 515-517

51. Vermulst M, Wanagat J, Kujoth GC et al (2008) DNA deletions and clonal mutations drive premature aging in mitochondrial mutator mice. Nat Genet 40, 392-394

52. Wallace DC (2005) A mitochondrial paradigm of metabolic and degenerative diseases, aging, and cancer: a dawn for evolutionary medicine. Annu Rev Genet 39, 359-407

53. Sarsour EH, Kalen AL, Goswami PC (2014) Manganese superoxide dismutase regulates a redox cycle within the cell cycle. Antioxid Redox Signal 20, 1618-1627

54. Ristow M (2014) Unraveling the truth about antioxidants: mitohormesis explains ROS-induced health benefits. Nat Med 20, 709-711
55. Sun N, Youle RJ, Finkel T (2016) The Mitochondrial Basis of Aging. Mol Cell 61, 654-666

56. Shadel GS, Horvath TL (2015) Mitochondrial ROS signaling in organismal homeostasis. Cell 163, 560-569

57. Lee SS, Lee RY, Fraser AG, Kamath RS, Ahringer J, Ruvkun G (2003) A systematic RNAi screen identifies a critical role for mitochondria in C. elegans longevity. Nat Genet 33, 40-48

58. Dillin A, Hsu AL, Arantes-Oliveira N et al (2002) Rates of behavior and aging specified by mitochondrial function during development. Science 298, 2398-2401

59. Zarse K, Schmeisser $S$, Groth $M$ et al (2012) Impaired insulin/IGF1 signaling extends life span by promoting mitochondrial L-proline catabolism to induce a transient ROS signal. Cell Metab 15, 451-465

60. Lee SJ, Hwang AB, Kenyon C (2010) Inhibition of respiration extends $C$. elegans life span via reactive oxygen species that increase HIF-1 activity. Curr Biol 20, 2131-2136

61. Munoz-Najar U, Sedivy JM (2011) Epigenetic control of aging. Antioxid Redox Signal 14, 241-259

62. Rando TA, Chang HY (2012) Aging, rejuvenation, and epigenetic reprogramming: resetting the aging clock. Cell 148, 46-57

63. Schroeder EA, Raimundo N, Shadel GS (2013) Epigenetic silencing mediates mitochondria stressinduced longevity. Cell Metab 17, 954-964

64. Shpilka T, Haynes CM (2018) The mitochondrial UPR: mechanisms, physiological functions and implications in ageing. Nat Rev Mol Cell Biol 19, 109-120

65. Nargund AM, Fiorese CJ, Pellegrino MW, Deng $P$, Haynes CM (2015) Mitochondrial and nuclear accumulation of the transcription factor ATFS-1 promotes OXPHOS recovery during the UPR $\mathrm{mt}$. Mol Cell 58, 123-133

66. Nargund AM, Pellegrino MW, Fiorese CJ, Baker BM, Haynes CM (2012) Mitochondrial import efficiency of ATFS-1 regulates mitochondrial UPR activation. Science $337,587-590$

67. Tian Y, Garcia G, Bian Q et al (2016) Mitochondrial Stress Induces Chromatin Reorganization to Promote Longevity and UPR(mt). Cell 165, 1197-1208

68. Felkai S, Ewbank JJ, Lemieux J, Labbé JC, Brown GG, Hekimi S (1999) CLK-1 controls respiration, behavior and aging in the nematode Caenorhabditis elegans. EMBO J 18, 1783-1792

69. Liu X, Jiang N, Hughes B, Bigras E, Shoubridge E, Hekimi S (2005) Evolutionary conservation of the clk-1-dependent mechanism of longevity: loss of mclk1 increases cellular fitness and lifespan in mice. Genes Dev 19, 2424-2434

70. Frezza C (2017) Mitochondrial metabolites: undercover signalling molecules. Interface Focus 7, 20160100

71. Sullivan LB, Gui DY, Vander Heiden MG (2016) Altered metabolite levels in cancer: implications for tumour biology and cancer therapy. Nat Rev Cancer 16, 680-693

72. Menzies KJ, Zhang H, Katsyuba E, Auwerx J (2016) Protein acetylation in metabolism-metabolites and cofactors. Nat Rev Endocrinol 12, 43-60 
73. Sutendra G, Kinnaird A, Dromparis P et al (2014) A nuclear pyruvate dehydrogenase complex is important for the generation of acetyl-CoA and histone acetylation. Cell 158, 84-97

74. Shi L, Tu BP (2015) Acetyl-CoA and the regulation of metabolism: mechanisms and consequences. Curr Opin Cell Biol 33, 125-131

75. Choudhary C, Weinert BT, Nishida Y, Verdin E, Mann M (2014) The growing landscape of lysine acetylation links metabolism and cell signalling. Nat Rev Mol Cell Biol 15, 536-550

76. Xie Z, Dai J, Dai L et al (2012) Lysine succinylation and lysine malonylation in histones. Mol Cell Proteomics 11, 100-107

77. Benayoun BA, Pollina EA, Brunet A (2015) Epigenetic regulation of ageing: linking environmental inputs to genomic stability. Nat Rev Mol Cell Biol 16, 593-610

78. Schultz MB, Sinclair DA (2016) Why NAD+ Declines during Aging: It's Destroyed. Cell Metab 23, 965-966

79. Camacho-Pereira J, Tarragó MG, Chini CCS et al (2016) CD38 Dictates Age-Related NAD Decline and Mitochondrial Dysfunction through an SIRT3-Dependent Mechanism. Cell Metab 23, 1127-1139

80. Imai S-I, Guarente L (2016) It takes two to tango: NAD+ and sirtuins in aging/longevity control. NPJ Aging Mech Dis 2, 16017

81. Gomes AP, Price NL, Ling AJ et al (2013) Declining $\mathrm{NAD}(+)$ induces a pseudohypoxic state disrupting nuclear-mitochondrial communication during aging. Cell $155,1624-1638$

82. Mouchiroud L, Houtkooper RH, Moullan N et al (2013) The NAD+/sirtuin pathway modulates longevity through activation of mitochondrial UPR and FOXO signaling. Cell 154, 430-441

83. Yoshino J, Mills KF, Yoon MJ, Imai S (2011) Nicotinamide mononucleotide, a key NAD+ intermediate, treats the pathophysiology of diet-and age-induced diabetes in mice. Cell Metab 14, 528-536

84. Quiros PM, Mottis A, Auwerx J (2016) Mitonuclear communication in homeostasis and stress. Nat Rev Mol Cell Biol 17, 213-226

85. Mammucari C, Gherardi G, Zamparo I et al (2015) The mitochondrial calcium uniporter controls skeletal muscle trophism in vivo. Cell Rep 10, 1269-1279

86. Franceschi $C$, Garagnani $P$, Parini $P$, Giuliani $C$, Santoro A (2018) Inflammaging: a new immune-metabolic viewpoint for age-related diseases. Nat Rev Endocrinol $14,576-590$

87. Davis BK, Wen H, Ting JP (2011) The inflammasome NLRs in immunity, inflammation, and associated diseases. Annu Rev Immunol 29, 707-735

88. Wenceslau CF, McCarthy CG, Szasz T et al (2014) Mitochondrial damage-associated molecular patterns and vascular function. Eur Heart J 35, 1172-1177

89. Zhang Q, Raoof M, Chen $Y$ et al (2010) Circulating mitochondrial DAMPs cause inflammatory responses to injury. Nature 464, 104-107

90. Grazioli S, Pugin J (2018) Mitochondrial DamageAssociated Molecular Patterns: From Inflammatory Signaling to Human Diseases. Front Immunol 9, 832
91. Pinti M, Cevenini E, Nasi M et al (2014) Circulating mitochondrial DNA increases with age and is a familiar trait: Implications for "inflamm-aging". Eur J Immunol 44, 1552-1562

92. Furman D, Chang J, Lartigue L et al (2017) Expression of specific inflammasome gene modules stratifies older individuals into two extreme clinical and immunological states. Nat Med 23, 174-184

93. Franceschi C, Garagnani P, Parini P, Giuliani C, Santoro A (2018) Inflammaging: a new immune-metabolic viewpoint for age-related diseases. Nat Rev Endocrinol $14,576-590$

94. Kim SJ, Xiao J, Wan J, Cohen P, Yen K (2017) Mitochondrially derived peptides as novel regulators of metabolism. J Physiol 595, 6613-6621

95. Hashimoto Y, Niikura T, Tajima $\mathrm{H}$ et al (2001) A rescue factor abolishing neuronal cell death by a wide spectrum of familial Alzheimer's disease genes and Abeta. Proc Natl Acad Sci U S A 98, 6336-6341

96. Ikonen M, Liu B, Hashimoto $Y$ et al (2003) Interaction between the Alzheimer's survival peptide humanin and insulin-like growth factor-binding protein 3 regulates cell survival and apoptosis. Proc Natl Acad Sci U S A 100, 13042-13047

97. Guo B, Zhai D, Cabezas E et al (2003) Humanin peptide suppresses apoptosis by interfering with Bax activation. Nature 423, 456-461

98. Cobb LJ, Lee C, Xiao J et al (2016) Naturally occurring mitochondrial-derived peptides are age-dependent regulators of apoptosis, insulin sensitivity, and inflammatory markers. Aging 8, 796-809

99. Zarse K, Ristow M (2015) A mitochondrially encoded hormone ameliorates obesity and insulin resistance. Cell Metab 21, 355-356

100. Lee C, Zeng J, Drew BG et al (2015) The mitochondrialderived peptide MOTS-c promotes metabolic homeostasis and reduces obesity and insulin resistance. Cell Metab 21, 443-454

101. Lee C, Kim KH, Cohen P (2016) MOTS-C: A novel mitochondrial-derived peptide regulating muscle and fat metabolism. Free Radic Biol Med 100, 182-187

102. Kim KH, Son JM, Benayoun BA, Lee C (2018) The Mitochondrial-Encoded Peptide MOTS-c Translocates to the Nucleus to Regulate Nuclear Gene Expression in Response to Metabolic Stress. Cell Metab 28, 516-524

103. Mangalhara KC, Shadel GS (2018) A MitochondrialDerived Peptide Exercises the Nuclear Option. Cell Metab 28, 330-331

104. Wong W (2018) Going nuclear with stress. Science Signaling 11, eaav4285

105. Muzumdar RH, Huffman DM, Atzmon G et al (2009) Humanin: a novel central regulator of peripheral insulin action. PLoS One 4, e6334

106. Lee C, Wan J, Miyazaki B et al (2014) IGF-I regulates the age-dependent signaling peptide humanin. Aging Cell 13, 958-961

107. Fuku N, Pareja-Galeano H, Zempo H et al (2015) The mitochondrial-derived peptide MOTS-c: a player in exceptional longevity? Aging Cell 14, 921-923

108. Zempo H, Fuku N, Nishida Y et al (2016) Relation 
between type 2 diabetes and $\mathrm{m}$. $1382 \mathrm{~A}>\mathrm{C}$ polymorphism which occurs amino acid replacement (K14Q) of mitochondria-derived MOTS-c. FASEB J 30, 956.1

109. Price NL, Gomes AP, Ling AJ et al (2012) SIRT1 is required for AMPK activation and the beneficial effects of resveratrol on mitochondrial function. Cell Metab 15, 675-690

110. Cantó C, Gerhart-Hines Z, Feige JN et al (2009) AMPK regulates energy expenditure by modulating NAD+ metabolism and SIRT1 activity. Nature 458, 1056-1060

111. Dunham-Snary KJ, Ballinger SW (2015) GENETICS. Mitochondrial-nuclear DNA mismatch matters. Science $349,1449-1450$

112. Rand DM (2017) Fishing for adaptive epistasis using mitonuclear interactions. PLoS Genet 13, e1006662

113. Tranah GJ (2011) Mitochondrial-nuclear epistasis: Implications for human aging and longevity. Ageing Res Rev 10, 238-252

114. McManus MJ, Picard M, Chen HW et al (2018) Mitochondrial DNA Variation Dictates Expressivity and Progression of Nuclear DNA Mutations Causing Cardiomyopathy. Cell Metab [Epub ahead of print]

115. Deuse T, Wang D, Stubbendorff $M$ et al (2015) SCNT-derived ESCs with mismatched mitochondria trigger an immune response in allogeneic hosts. Cell Stem Cell 16, 33-38

116. Betancourt AM, King AL, Fetterman JL et al (2014) Mitochondrial-nuclear genome interactions in non-alcoholic fatty liver disease in mice. Biochemical J 461, 223-232

117. Fetterman JL, Zelickson BR, Johnson LW et al (2013) Mitochondrial genetic background modulates bioenergetics and susceptibility to acute cardiac volume overload. Biochemical J 455, 157-167

118. Raimundo N, Kriško A (2018) Cross-organelle communication at the core of longevity. Aging 10, 15-16

119. Rieusset J (2018) The role of endoplasmic reticulummitochondria contact sites in the control of glucose homeostasis: an update. Cell Death Dis 9, 388

120. Janikiewicz J, Szymański J, Malinska D et al (2018) Mitochondria-associated membranes in aging and senescence: structure, function, and dynamics. Cell Death Dis 9, 332

121. Wang $\mathrm{CH}$, Chen $\mathrm{YF}, \mathrm{Wu} \mathrm{CY}$ et al (2014) Cisd2 modulates the differentiation and functioning of adipocytes by regulating intracellular $\mathrm{Ca} 2+$ homeostasis. Hum Mol Genet 23, 4770-4785

122. Chen YF, Kao CH, Chen YT et al (2009) Cisd2 deficiency drives premature aging and causes mitochondriamediated defects in mice. Genes Dev 23, 1183-1194

123. Titorenko VI, Terlecky SR (2011) Peroxisome metabolism and cellular aging. Traffic 12, 252-259

124. Sebastián D, Palacín M, Zorzano A (2017) Mitochondrial dynamics: coupling mitochondrial fitness with healthy aging. Trends Mol Med 23, 201-215

125. Koepke JI, Nakrieko KA, Wood CS et al (2007) Restoration of peroxisomal catalase import in a model of human cellular aging. Traffic 8, 1590-1600

126. Santos MJ, Quintanilla RA, Toro A et al (2005) Peroxisomal proliferation protects from $\beta$-amyloid neurodegeneration. J Biol Chem 280, 41057-41068

127. Nell HJ, Au JL, Giordano CR et al (2017) Targeted Antioxidant, Catalase-SKL, Reduces Beta-Amyloid Toxicity in the Rat Brain. Brain Pathol 27, 86-94

128. Yoboue ED, Sitia R, Simmen T (2018) Redox crosstalk at endoplasmic reticulum (ER) membrane contact sites (MCS) uses toxic waste to deliver messages. Cell Death Dis 9, 331

129. Carmona-Gutierrez D, Hughes AL, Madeo F, Ruckenstuhl C (2016) The crucial impact of lysosomes in aging and longevity. Ageing Res Rev 32, 2-12

130. Soto-Heredero G, Baixauli F, Mittelbrunn M (2017) Interorganelle communication between mitochondria and the endolysosomal system. Front Cell Dev Biol 5, 95

131. Hughes AL, Gottschling DE (2012) An early age increase in vacuolar $\mathrm{pH}$ limits mitochondrial function and lifespan in yeast. Nature 492, 261-265

132. Elbaz-Alon Y, Rosenfeld-Gur E, Shinder V, Futerman AH, Geiger T, Schuldiner M (2014) A dynamic interface between vacuoles and mitochondria in yeast. Dev Cell 30, 95-102

133. Klecker T, Westermann B (2014) Mitochondria Are Clamped to Vacuoles for Lipid Transport. Dev Cell 30, $1-2$

134. Murley A, Sarsam RD, Toulmay A, Yamada J, Prinz WA, Nunnari J (2015) Ltc1 is an ER-localized sterol transporter and a component of ER-mitochondria and ER-vacuole contacts. J Cell Biol 209, 539-548

135. Hönscher C, Mari M, Auffarth K et al (2014) Cellular metabolism regulates contact sites between vacuoles and mitochondria. Dev Cell 30, 86-94

136. Durieux J, Wolff S, Dillin A (2011) The cell-nonautonomous nature of electron transport chain-mediated longevity. Cell 144, 79-91

137. Woo DK, Shadel GS (2011) Mitochondrial stress signals revise an old aging theory. Cell 144, 11-12

138. Zhang Q, Wu X, Chen P et al (2018) The mitochondrial unfolded protein response is mediated cell-nonautonomously by retromer-dependent Wnt signaling. Cell 174, 870-883.e817

139. Owusu-Ansah E, Song W, Perrimon N (2013) Muscle mitohormesis promotes longevity via systemic repression of insulin signaling. Cell 155, 699-712

140. Kim KH, Jeong YT, Oh H et al (2012) Autophagy deficiency leads to protection from obesity and insulin resistance by inducing Fgf21 as a mitokine. Nat Med 19, 83-92

141. Berendzen KM, Durieux J, Shao LW et al (2016) Neuroendocrine coordination of mitochondrial stress signaling and proteostasis. Cell 166, 1553-1563.e1510

142. Shao L-W, Niu R, Liu Y (2016) Neuropeptide signals cell non-autonomous mitochondrial unfolded protein response. Cell Res 26, 1182-1196

143. da Cunha FM, Torelli NQ, Kowaltowski AJ (2015) Mitochondrial Retrograde Signaling: Triggers, Pathways, and Outcomes. Oxid Med Cell Longev 2015, 482582

144. Lee C, Kim KH, Cohen P (2016) MOTS-c: a novel mitochondrial-derived peptide regulating muscle and fat metabolism. Free Radic Biol Med 100, 182-187

145. Bachar AR, Scheffer L, Schroeder AS et al (2010) 
Humanin is expressed in human vascular walls and has a cytoprotective effect against oxidized LDL-induced oxidative stress. Cardiovasc Res 88, 360-366

146. Guevara-Aguirre J, Balasubramanian $\mathrm{P}$, Guevara-Aguirre $M$ et al (2011) Growth hormone receptor deficiency is associated with a major reduction in pro-aging signaling, cancer, and diabetes in humans. Sci Transl Med 3, 70ra13

147. Cobb LJ, Lee C, Xiao J et al (2016) Naturally occurring mitochondrial-derived peptides are age-dependent regulators of apoptosis, insulin sensitivity, and inflammatory markers. Aging 8, 796-809 